Journal of

Cancer Research and Therapeutic Oncology

JScholar

\title{
Post-occlusive Reactive Hyperemia in Mouse Melanoma
}

\author{
Julien Reyal ${ }^{1, *}$, Nicolas Lebas ${ }^{2}$, Ivana Zlatanova ${ }^{1}$, José Vilar ${ }^{1}$, Jean-Sébastien Silvestre ${ }^{1}$ \\ ${ }^{1}$ Paris Cardiovascular Research Center, INSERM U970, Hôpital Européen Georges Pompidou, Paris-Descartes Uni- \\ versity, Paris, France \\ ${ }^{2}$ Department of Biostatistics, Institut Curie, Paris, France \\ ${ }^{*}$ Corresponding author: Dr. Julien Reyal, PARCC-Inserm U970, Université Paris Descartes, 56 Rue Leblanc, 75015 \\ Paris, France. Email: julien.reyal@gmail.com; Tel: +331539880 60; Fax: +33153987951
}

Received Date: December 07, 2014; Accepted Date: April 27, 2015; Published Date: April 29, 2015

Citation: Julien Reyal, et al. (2015) Post-occlusive Reactive Hyperemia in Mouse Melanoma. J Cancer Res Therap Oncol 1: 1-6

\begin{abstract}
Post-occlusive reactive hyperemia (PORH) is a physiological reaction characterizing the vascular reactivity to application and cessation of blood flow occlusion. Increasing evidence indicates that the PORH may reduce tumor hypoxia and could be used as a clinically relevant method to improve the efficiency of conventional anti-cancer therapies. In order to experiment this new concept, we developed a simple and reproducible mouse model of skin PORH using a leg tourniquet to produce temporary vascular occlusion. Footpad superficial perfusion was continuously measured by laser speckle contrast imaging. We then analyzed the incidence of PORH on B16-F10 melanomas cells injected in the footpad skin. The mouse model reproduced the characteristics of the skin PORH in humans, with a close relationship between the duration of the vascular occlusion and the hyperemia amplitude as well as extent. We also unravelled that PORH also occurred in growing melanoma with a significant $48 \%$ median hyperemia after three minutes of vascular occlusion. We therefore described for the first time a PORH phenomenon in a locally very advanced and necrotic murine melanoma that may pave the way for the development of complementary therapeutic approaches to dampen the growth of aggressive tumors.
\end{abstract}

Keywords: Laser speckle contrast imaging; Limb tourniquet; Murine melanoma; Post-occlusive reactive hyperemia; Tumor hypoxia

\section{Introduction}

Tumor angiogenesis [1] leads to pathological, dilated and tortuous vascular network $[2,3]$, that causes abnormal perfusion of the hypoxic tumor area $[4,5]$, leading to, at least in part, resistance to conventional anti-cancer therapies [6-8].

Post-occlusive reactive hyperemia (PORH) is an ubiquitous physiological vascular reaction $[9,10]$, first described in the skin [11], that consists of a hyperemia peak, occurring just after the release of a temporary vascular occlusion, followed by a progressive return to the pre-occlusion perfusion level. Since tumor blood vessels are mostly functional and sensitive to vasoactive agents [12-14], one can speculate that PORH could increase tumor perfusion. In this line, PORH has been recently described as a mean to enhance temporary and locally the tumor perfusion and oxygenation levels thus increasing the outcome of anticancer therapies in human [15]. Tumor PORH has also been recently demonstrated in human basal cell carcinoma [16].

(C)2015 The Authors. Published by the JScholar under the terms of the Creative Commons Attribution License http://creativecommons.org/licenses/ by $/ 3.0 /$, which permits unrestricted use, provided the original author and source are credited.
In the present study, we set up a simple and reproducible mouse model of leg PORH to test the effect of vascular occlusion on a mouse syngenic B16 melanoma perfusion [17]. In addition, we used the laser speckle contrast imaging (LSCI), a non-invasive and accurate technology to assess skin blood flow [18]. LSCI may provide a valuable tool to develop our knowledge of tumor PORH phenomenon and therapeutic potential.

\section{Material and Methods}

\section{Mice characteristics}

All experiments were conducted according to the local and French veterinary guidelines and those formulated by the European Community for experimental animal use (No. 07430). 8 weeks-old C57BL/6 mice were obtained from Janvier labs (France). Syngeneic tumor cell line B16-F10-luc was cultured in DMEM supplemented with $10 \%$ fetal calf serum, $1 \%$ antibiotics (penicillin and streptomycin), and $2 \mathrm{mM}$ glutamine in humidified incubator with $5 \% \mathrm{CO}_{2}$ and $95 \%$ air.

\section{Anesthesia}

Mice were anesthetized using $1.5 \%$ isoflurane, since this 
anesthetic induces a modest effect on tumor perfusion and oxygenation [19]. It also ensures a perfect anesthesia without perturbing motion during laser speckle measurement. Air was used with a constant flow of $2 \mathrm{~L} / \mathrm{min}$.

\section{Footpad tumor implantation (Figure1)}

Melanoma cells were harvested by trypsination and $10^{6} \mathrm{~B} 16-$ F10-luc cells were suspended in 10 microliters of PBS. 10 microliters per mice were then injected into the footpad of the left leg of 10 anesthetized mice, using insulin needle (29G) oriented towards the toes and inducing intradermal bubble in the hairless area of the footpad. Tumor growth was observed every three days. Tumor sizes were measured with an electronic caliper. Laser speckle experiments were performed at 14 days after the injection to allow easy and reproducible measurement of tumor $\mathrm{PO}_{2}$ using fluorescence quenching oximetry probe (Oxford Optronics).
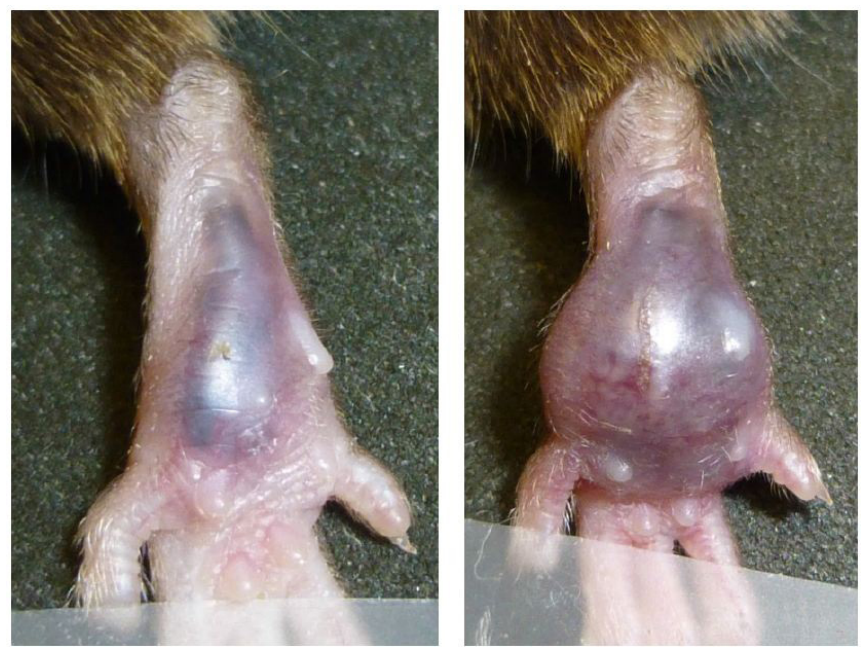

Figure 1: Representative photomicrographs of foot-pad B16 melanoma, 7 (left) and 14 (Right) days after injection.

\section{Laser Speckle Contrast Imaging (LSCI) and condi- tions for data acquisition (Figure 2)}

Cutaneous blood perfusion was recorded using a Laser Speckle Contrast imaging system (PERICAM PSI system, high resolution model, Perimed). The laser wavelength was $785 \mathrm{~nm}$. The laser head was positioned $10 \mathrm{~cm}$ above the skin and remained perfectly motionless thanks to its fixing system onto the experiment bench. The acquisition rate was 2 frames per second. The temperature of the experimental room was monitored at $22^{\circ} \mathrm{C}$. Anesthetized mice were maintained in a decubitus position on a laser speckle recording black carpet (Perimed). Limbs were fixed with transparent tape, the left leg being fixed in extension to allow its introduction into a lake tissue vascular surgical tourniquet (Argyle vascular tourniquet, with a surgical needle holder to tighten the tourniquet). The tail was attached to the right side of the mouse, in order to avoid the field of laser recording. Five minutes were required between the beginning of anesthesia and that of data acquisition. Record of laser speckle signal of the left leg required a perfect immobility of the animal that was well-achieved using isoflurane anesthesia.

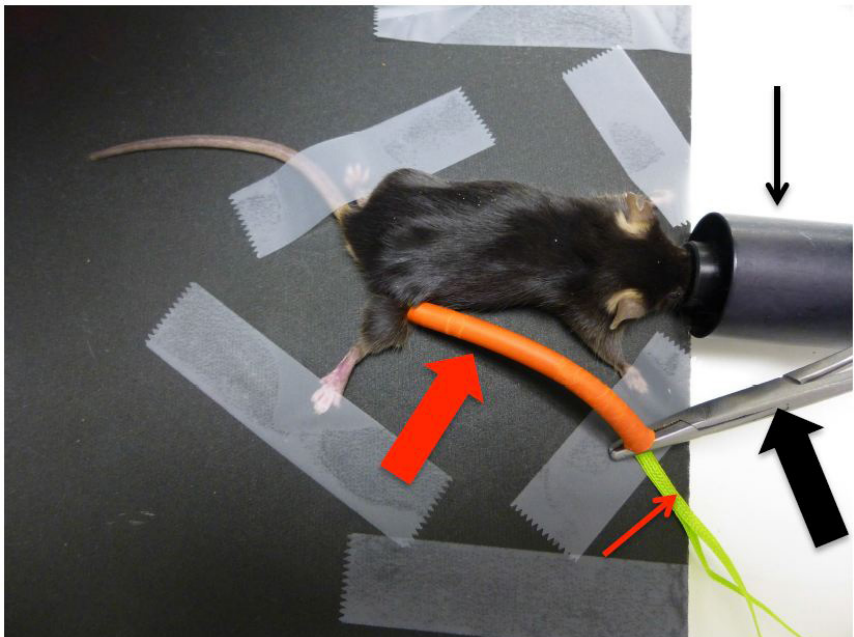

Figure 2: Photography of the surgical vascular tourniquet system. Right leg occlusion photography issued from preliminary experiments. Only left side was used for definitive experiment. Surgical needle holder permitted to tighten the tourniquet around the leg basis. Thick red arrow reveals the vascular tourniquet silicone external part, which is tighten by a surgical needle holder (thick black arrow), clamped on tissue lac (thin red arrow). Thus, leg perfusion was totally occluded at the leg basis. Thin black arrow represents the isoflurane nose.

Measurements of Melanoma oxygen partial pressure

Fluorescence quenching oximetry probe measured the tumor oxygen partial pressure (reference BF/ OT/E, Oxylite system, Oxford Optronics) [20] and was placed into the tumor center through a $21 \mathrm{G}$ catheter.

\section{Experimental Protocols}

1. Observation of perfusion in footpad healthy skin without vascular occlusion: Ten mice were used. Skin perfusion was evaluated without warming pad to avoid any detrimental effect on skin blood flow during a long period of observation. We first studied the skin perfusion of the left footpad for $20 \mathrm{~min}$ utes. We also measured rectal temperature at the beginning of the experiment, and after 20 minutes of observation.

2. Post- occlusive reactive hyperemia study in footpad healthy skin: In a second step, we studied the healthy skin reaction to leg vascular occlusion through tourniquet, and the influence of the occlusion time ( 30 seconds or 3 minutes of occlusion) on the characteristics of PORH assessed by laser speckle contrast imaging. After placing the mouse on the black carpet, laser speckle recording was started, and a basic reference measure was obtained in the first 30 seconds. Then, vascular occlusion of the left leg was performed at the groin through the surgical tourniquet. The arrest of perfusion was controlled directly by LSCI. Unclamping was accompanied by immediate reperfusion. The perfusion was then measured until return to stable levels. 30 seconds and 3 minutes occlusion were realized on the same mice, with one day interval. The same group of 10 mice then received injection of B16 melanoma cells in the left footpad.

3. Observation of perfusion in footpad B16 melanoma without vascular occlusion: Fourteen days after the injection, we 
observed the melanoma perfusion during 20 minutes, to identify the ideal timing of vascular occlusion induction. The ideal moment is the time corresponding to the hypo-perfused state of the melanoma in order to have a less vasodilated state of tumor vasculature. This allowed us to reveal the maximal potential of vasodilatation induced by PORH. A drop in tumor perfusion was observed during the first 5 minutes leading to set the time of vascular occlusion at 5 minutes after the start of the laser speckle recording.

4. Post- occlusive reactive hyperemia study in footpad B16 melanoma: Next, vascular occlusion was induced for $3 \mathrm{~min}$ utes, since this duration has been shown to induce a marked hyperemia reaction in healthy skin.
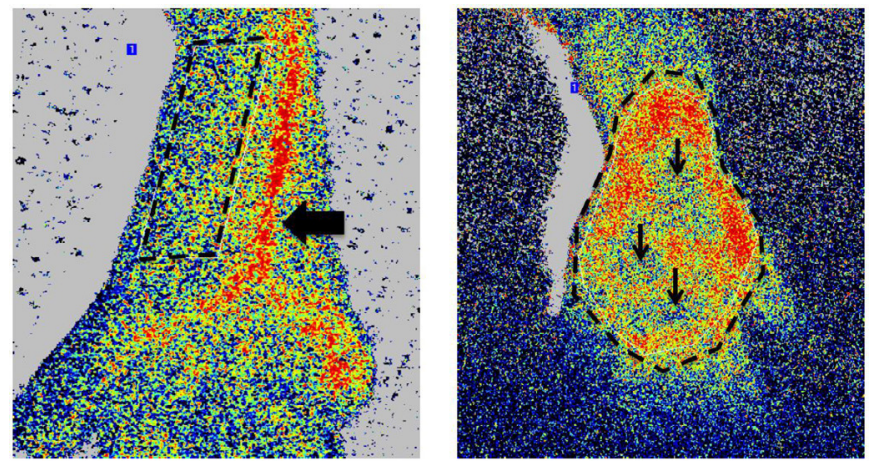

Figure 3: Laser speckle contrast images of healthy skin (A) and melanoma (B) after 14 days. Dotted line represents the corresponding ROI. Tumor ROI were generally very well perfused in the periphery, and sometimes displayed central hypoperfusion zones (Thin black arrows). Black thick arrow reveals the foot-pad vessels. Foot-pad vessels disappeared at 14 days, i.e. at the time of the laser speckle contrast imaging measurement.

5. Melanoma oxygen partial pressure (PO2) and tumor size caliper measurement: At the end of the LSCI recording, steady tumor PO2 $(\mathrm{mmHg})$ was measured 10 minutes after the introduction of oxylite system oximetry probe in the tumor center in one point. Then, tumor size was measured by caliper electronic and tumor volume $(\mathrm{mm} 3)$ calculated using the formula of an ellipsoid tumor volume $\left(\mathrm{V}=\mathrm{L} \mathrm{x} \mathrm{l}^{2} \times 0.52\right)$. Mice were then sacrificed and footpad tumors were opened with surgical instruments.

\section{LSCA Data analysis (Figure 3)}

Data were digitalized, stored on a computer and analyzed offline with a specific analysis software (Pimsoft, Perimed). Perfusion was expressed as perfusion unit (Perfusion Unit, PU).

i. Selection of Region Of Interest (ROI): Healthy skin ROI is the region of the footpad located externally to the vessels of the leg and was designed as a quadrilateral shape starting near knee and ended at the bifurcation of the footpad vessels. Healthy skin ROI was selected in mice without tumor only. Tumor ROI appeared as hyper-perfused area, at least in the periphery, and sometimes with central hypoperfused necrotic area. Tumor ROI was drawn as a free form.

ii. Selection of Time Of Interest (TOI) periods, and description of post- occlusive reactive hyperemia data: All TOI periods lasted 10 seconds and corresponded to stable ROI. TOI were placed every 5 minutes. Basal pre-occlusion TOI was determined just before the beginning of the vascular clamping.
Hyperemia peak TOI was centered at the time of the hyperemia peak. Hyperemia percentage was calculated as: hyperemia peak - pre-occlusion value/pre-occlusion value. The value of tumor perfusion always returned to its pre-occlusion level. The time to peak was the time in seconds between the occlusion release and the hyperemia peak. The hyperemia duration corresponds to the time between occlusion release and the return to the pre-occlusion perfusion value. The repayment / debt ratio was the ratio of the AUC (Area Under Curve) of the post-occlusive hyperemia TOI and the AUC of perfusion debt during vascular occlusion.

\section{Statistical analysis}

All analyses were performed with R software. We used MannWhitney and Kruskall-Wallis tests to compare the medians between two groups or more. Wilcoxon's matched pairs, signed ranks and Friedman's tests were used for paired comparisons. Correlations between the entire tumor's percentage and variables of interest were assessed with Spearman correlation test. Significance was accepted at $\mathrm{p}<0.05$. Results are expressed as the median and interquartile in parentheses.

\section{Results}

Footpad skin perfusion was stable during 20 min recording even without warming pad (Figure 4, Table 1)

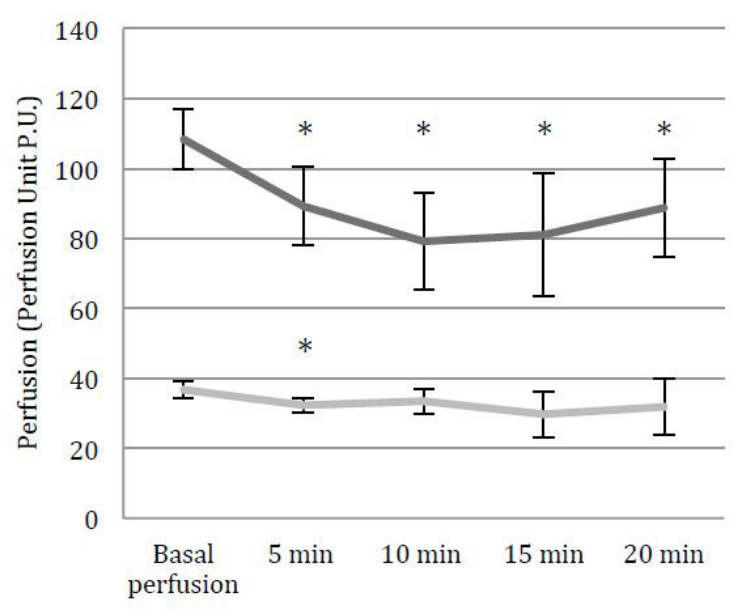

Figure 4: Evolution of median healthy skin and melanoma perfusion over $20 \mathrm{~min}$ of laser speckle contrast imaging measurement. ${ }^{*} \mathrm{p}<0.05$, vertical bar indicates interquartile. Black line represents melanoma perfusion, grey line healthy skin perfusion.

\begin{tabular}{|l|l|l|l|l|l|}
\hline & $\begin{array}{l}\text { Basal per- } \\
\text { fusion }\end{array}$ & $\mathbf{5}$ min & $\mathbf{1 0 ~}$ min & 15 min & 20 min \\
\hline Melanoma & $\begin{array}{l}108.36 \\
(17.25)\end{array}$ & $\begin{array}{l}89.18 \\
(22.48)^{*}\end{array}$ & $\begin{array}{l}79.11 \\
(27.44)^{*}\end{array}$ & $\begin{array}{l}81.09 \\
(35.38)^{*}\end{array}$ & $\begin{array}{l}88.73 \\
(28.3)^{*}\end{array}$ \\
\hline $\begin{array}{l}\text { Healthy } \\
\text { Skin }\end{array}$ & $\begin{array}{l}36,85 \\
(4.98)\end{array}$ & $\begin{array}{l}32,25 \\
(4.38)^{*}\end{array}$ & $\begin{array}{l}33,45 \\
(7.1)\end{array}$ & $\begin{array}{l}29,65 \\
(12.83)\end{array}$ & $\begin{array}{l}31,95 \\
(15.93)\end{array}$ \\
\hline
\end{tabular}

Table 1: Quantitative analysis of Laser speckle contrast imaging perfusion of healthy skin and melanoma over 20 minutes of continuous measurement. ${ }^{*} \mathrm{p}<0.05$ versus basal perfusion. Values are expressed as median values (interquartile). 
Skin perfusion was stable over 20 minutes, with no significant difference between the basal perfusion and the end of measurement $(\mathrm{p}=0.77)$. However, there was a fall in perfusion recording at the onset of recording (from $36.85 \mathrm{PU}$ (5) at 0 minutes, to $32.25 \mathrm{PU}(4.4)$ at 5 minutes) $(\mathrm{p}=0.006)$, and a rise of the perfusion at the end of recording (from 29.65 $\mathrm{PU}(12.8)$ at 15 minutes, to $31.95 \mathrm{PU}(15.9)$ at 20 minutes $)(\mathrm{p}=0.002)$. The initial temperature of $34.4^{\circ} \mathrm{C}(0,8)$ dropped to $27.8^{\circ} \mathrm{C}(1.5)$ in 20 minutes, with consequent loss of $7.1^{\circ} \mathrm{C}(2.5)(\mathrm{p}=0.002)$, in contrast to the robust preservation of the footpad skin perfusion during 20 minutes. Therefore, absence of warming pad during experiments did not markedly impact the basal skin perfusion in our experimental conditions.

Post-occlusive reactive hyperemia values increased with vascular occlusion duration, as in human skin PORH (Figure 5, Table 2)



Figure 5: Boxplot graphic of healthy skin and melanoma hyperemia percentage secondary to 30 seconds and 3 minutes of vascular occlusion. All groups have mutually statistical significant difference compared to 3 minutes occlusion in healthy skin group $(\mathrm{p}<0.05)$.

\begin{tabular}{|l|l|l|l|}
\hline & \multicolumn{2}{|c|}{ Healthy Skin } & \multicolumn{1}{|c|}{ Melanoma } \\
\hline & $\begin{array}{l}30 \text { seconds } \\
\text { occlusion }\end{array}$ & $\begin{array}{l}3 \text { minutes } \\
\text { occlusion }\end{array}$ & $\begin{array}{l}3 \text { minutes } \\
\text { occlusion }\end{array}$ \\
\hline ROI surface (mm2) & $10(2.2)$ & $9.4(1.1)$ & $29.9(5.7)^{*}$ \\
\hline Basal perfusion (PU) & $35.1(4.6)$ & $32(5)$ & $81.5(33.3)^{*}$ \\
\hline Peak hyperemia (PU) & $74.5(6.1)$ & $78.8(24.3)$ & $131.7(71.4)^{\star}$ \\
\hline Hyperemia percentage (\%) & $105(24)^{\star}$ & $160(42.4)$ & $48(43.3)^{\star}$ \\
\hline Time to peak (secondes) & $16.5(3.3)^{\star}$ & $24.5(8.3)$ & $46.5(26.8)^{*}$ \\
\hline $\begin{array}{l}\text { Hyperemia duration (sec- } \\
\text { ondes) }\end{array}$ & $46.5(14.8)^{*}$ & $146(41)$ & $248.5(67.5)^{\star}$ \\
\hline Repayment/debt ratio (\%) & $229.6(76.9)^{*}$ & $125.7(37.3)$ & $143(47.5)^{*}$ \\
\hline
\end{tabular}

Table 2: Quantitative analysis of laser speckle contrast imaging perfusion of healthy skin and B16 melanoma post-occlusive reactive hyperemia $\left({ }^{*} \mathrm{p}<0.05\right.$ versus 3 minutes occlusion in healthy skin). Values are expressed as median values (interquartile).

Healthy footpad skin ROI size $(\mathrm{p}=0.38)$ and basal perfusion $(\mathrm{p}=0.12)$ were equivalent before 30 seconds or 3 minutes of occlusion. PORH values increased between 30 seconds and 3 minutes of occlusion. Hence, hyperemia percentage raised strongly from $105 \%(24)$ to $160 \%(42.4)(\mathrm{p}=0.002)$. Hyperemia peak increased from 74.5 PU (6.1) to $78.8 \mathrm{PU}$ (24.3) but did not reach statistical significance $(\mathrm{p}=0.09)$. Time to peak also markedly increased from 16.5 seconds (3.3) to 24.5 seconds (8.3) $(\mathrm{p}=0.006)$ as well as hyperemia duration that was enhanced from 46.5 seconds (14.8) to 146 seconds (41) $(\mathrm{p}=0.0002)$. As expected, the repayment/debt ratio decreased between 30 seconds and 3 minutes of occlusion, from $229.6 \%$ (76.9) to $125.7 \%$ (37.3) ( $\mathrm{p}=0.0007)$, respectively. Indeed, perfusion debt increased much more (1048.8 to 5875.2) than perfusion repayment (2476 to 7092) according to the duration of occlusion duration. Thus, like in human, PORH occurred in mouse skin and the intensity and the duration of hyperemia was related to the duration of occlusion $[9,10]$.

Melanoma perfusion was higher than that of healthy skin and decreased during the first 5 minutes of LSCA measurement (Figure 4)

Tumor perfusion was higher than that of healthy skin with $\mathrm{PU}$ of $108.4(17.2)$ versus $40(6.6)(\mathrm{p}=0.002)$, respectively, revealing the intense melanoma tumor angiogenesis [21] (Table 1, Figure5). Tumor perfusion decreased rapidly and significantly from 108.4 PU (17.2) to $89.2 \mathrm{PU}(22.5)(\mathrm{p}=0.002)$ after 5 minutes of recording and reached $88.7 \mathrm{PU}(28.3)$ at 20 minutes (p $=0.03$ versus $\mathrm{T} 0$ ). This drop may reflect the well-know tumor perfusion instability, particularly during anesthesia $[22,23]$. There was no significant difference in tumor perfusion between 5 minutes and 10 minutes $(p=0.084)$. Therefore, we have decided to wait only 5 minutes after the start of tumor perfusion recording to induce vascular occlusion in order to have the less dilated tumor micro-vascular network before occlusion and to avoid negative impact on tumor PORH amplitude.

Post-occlusive reactive hyperemia occurred in melanoma, with different characteristics compared to healthy skin (Figure 6, Table 2)

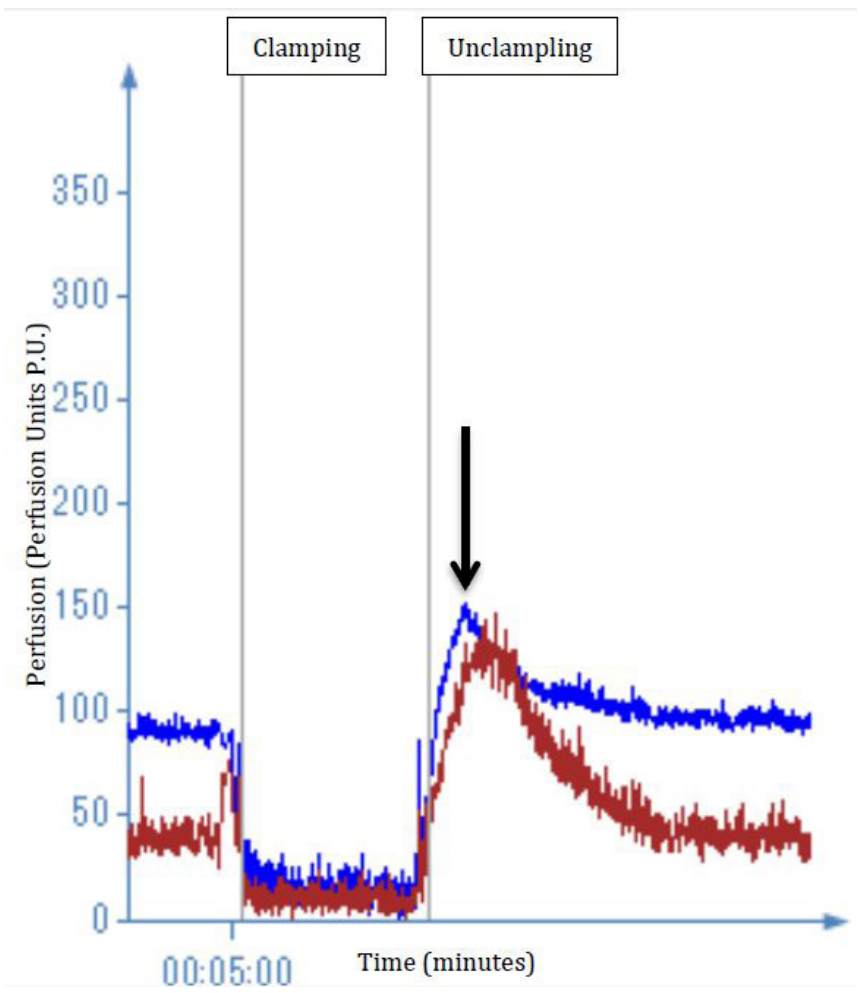

Figure 6: One B16 Melanoma perfusion curve (blue) issued from laser speckle contrast imaging software, compared to healthy skin perfusion (red). Tumor hyperemia peak is showed by a black arrow. Melanoma basal perfusion is higher than healthy skin, but hyperemia percentage is weaker, secondary to 3 minutes of vascular occlusion. 
Post -occlusive hyperemia was found in each tumor. The median percentage of hyperemia was $48 \%$ (43.4) \% versus $160 \%$ (42.4) for healthy skin $(\mathrm{p}=0.001)$. All data were different between the tumor and the healthy skin, revealing a particular vascular reactivity in the melanoma. Peak hyperemia was higher (PU 131.7 (71.4) versus 78.8 PU (24.3), p =0.02), the time to peak was longer (46.5 seconds (26.8) versus 24.5 seconds (8.3), $\mathrm{p}<0.001$ ) as well as the duration of hyperemia (248.5 seconds (67.5) versus 146 seconds (41), $\mathrm{p}=0.001)$ in tumor versus healthy skin, respectively. Concomitantly to the increase of the duration of hyperemia, the repayment/debt ratio was higher in the tumor when compared to that of healthy skin (143\% (47.5) versus $125.7 \%$ (37.3), $\mathrm{p}=0.04$, respectively). Secondary to the 3 minutes vascular occlusion, tumor vasodilatation was thus feebler (with a weaker PORH percentage), but longer than in healthy skin vessels (thus with a lengthier hyperemia).

\section{Melanoma volume correlated negatively with post-occlusive reactive hyperemia (Figure 7) \\ correlation between the entire tumor's percentage of hyperemia and volume}

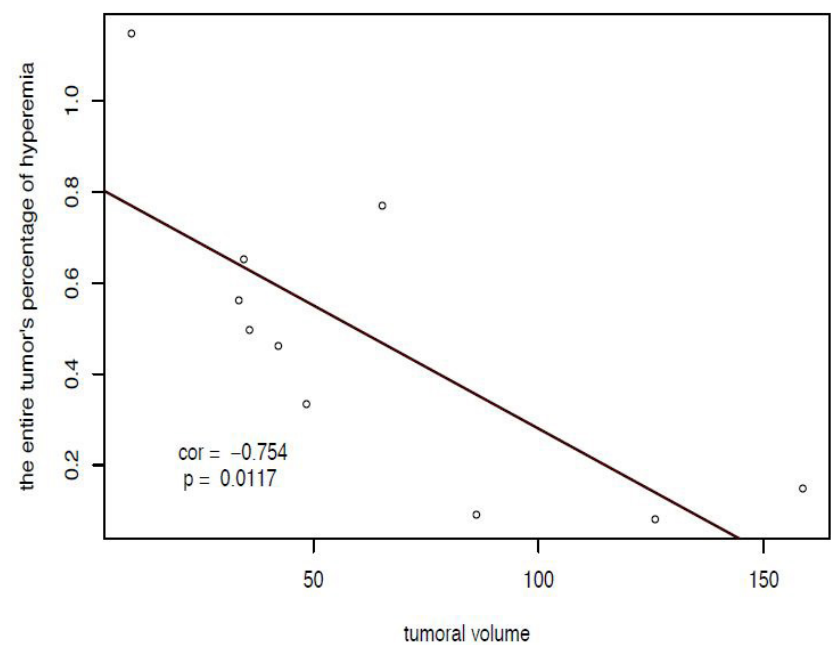

Figure 7: Negative correlation curve between melanoma hyperemia percentage and melanoma volume. Smallest melanoma had the highest hyperemia percentage (superior to 100\%), compared to the biggest melanoma that showed a hyperemia percentage inferior to $20 \%$.

Tumor volume $\left(45.3 \mathrm{~mm}^{3}(46.1)\right)$ and surface $\left(29.8 \mathrm{~mm}^{2}\right.$ (16.6)) were negatively correlated with the hyperemia percentage $(\mathrm{r}=-0.75, \mathrm{p}=0.01$ and $\mathrm{r}=-0.83, \mathrm{p}=0.003$, respectively). Central necrosis is generally proportional to tumor growth and reflected the degree of aggressive tumor. It is likely that necrosis also reduced the vasoactive potential of a tumor, dampening the PORH phenomenon. Accordingly, the melanoma were strongly anoxic and necrotic, with a median $\mathrm{PO}_{2}$ of $0.75 \mathrm{mmHg}(10)$, and a visual aspect of black soft necrosis during tumor opening after the sacrifice of the mice.

\section{Discussion}

In the present study, we have developed a simple and reliable mouse model of footpad PORH using a surgical tourniquet and a laser speckle contrast imaging system. Even without warming pad, the healthy skin perfusion remained relatively steady. Such mouse model of PORH displays the same characteristics than that of human skin or others organs $(9,10)$.
Hence, our mouse model may constitute a guileless and useful replica of skin PORH that may be of high interest for further analysis of molecular and cellular mechanisms of PORH. Interestingly, we also created a footpad B16 melanoma model. Implantation was always successful (10/10), with rapid growth over 14 days. Without the need to shave the footpad, melanoma perfusion was directly assessed with laser speckle contrast imaging, allowing a direct and easy measure of PORH in melanoma through a non-invasive way. Footpad constitutes a very useful location in order to test the effect of PORH on different type of murine or xenograft-derived cell lines, especially during radiotherapy and/or chemotherapy treatment.

This model will also permit to assess the diagnostic value of tumor vascular reactivity to temporary vascular occlusion, since tumor vascular immaturity is often related to tumor aggressiveness[24]. Accordingly, in our study, melanoma hyperemia percentage was inversely correlated to melanoma size $(\mathrm{p}=0.01)$, that is linked to aggressive state of the tumor. This study is also the first demonstration of the existence of PORH in an aggressive malignant tumor. Furthermore, it was the first demonstration of a significant PORH in a melanoma with marked central necrotic area suggesting that hyperemia can occur in aggressive tumor and may cause increases in perfusion and oxygenation in the peripheral zone of the tumor. Interestingly, such area display high resistance to radiotherapy and/or chemotherapy treatments $[6,8]$. However, the lacks of tools required to study spatially, in a hypoxic zone, the rapid variations of perfusion and oxygenation that occur during PORH dampen research on this specific topic.

In our experimental conditions, PORH in B16 melanoma was different from that of healthy skin suggesting that the vessels irrigating the tumor have a passive or active role in tumor PORH phenomenon. In correlation with results obtained after laser speckle contrast imaging, extensive study of tumor vessels architecture and network [25] (including micro-vessels density, vessels diameter, vascular surface or pericyte coverage), as well as intravital vascular imaging at different time of tumor growth will be useful to understand the respective role of intrinsic or extrinsic tumor vessels.

One can then speculate that post-occlusive reactive hyperemia may become an useful clinical method $[15,16]$, not only in patients with arm or leg tumors as illustrated by the mouse footpad melanoma model, but also in internal organs tumors. Indeed, the application of intravascular temporal occlusion of tumor vascular supply through balloon catheters technology (or perivascular occlusion with a vascular occluder) may increase solid malignant tumors perfusion and oxygenation during chemotherapy or radiotherapy, and, subsequently treatment efficiency.

\section{Conclusion}

We have developed a simple and reproducible murine model of skin PORH using laser speckle contrast imaging technology that will foster further analysis of PORH in healthy footpad skin. Significant PORH was also demonstrated in B16 melanoma with a median hyperemia percentage of $48 \%$ at the surface of necrotic tumors, suggesting a putative therapeutic poten- 
tial, even in locally advanced tumors. Additional experiments using laser speckle contrast imaging, histological and intravital imaging are required to confirm and develop our results.

\section{Conflict of Interest and Disclosure Statement}

Julien Reyal has filed a patent in cooperation with the Agency for Medical Innovations (GmbH, A.M.I., Austria) in 2010, and is registered as a co-inventor of this patent, describing a portal vein vascular occluder for another application that could eventually be modified and used clinically for tumoral PORH (Patent Application WO/2010/102661). Others authors did not have any conflict of interest.

\section{Acknowledgements}

Julien Reyal was supported by a Master grant from the Fondation pour la Recherche Médicale (FRM).

\section{References}

1) Folkman J (1990) What is the evidence that tumors are angiogenesis dependent? J Natl Cancer Inst 82: 4-6.

2) Less JR, Skalak TC, Sevick EM, Jain RK (1991) Microvascular architecture in a mammary carcinoma: branching patterns and vessel dimensions. Cancer Res 51: 265-273.

3) Sharma S, Sharma MC, Sarkar C (2005) Morphology of angiogenesis in human cancer: a conceptual overview, histoprognostic perspective and significance of neoangiogenesis. Histopathology 46: 481-489.

4) Kimura H, Braun RD, Ong ET, Hsu R, Secomb TW, et al. (1996) Fluctuations in red cell flux in tumor microvessels can lead to temporary hypoxia and reoxygenation in tumor parenchyma. Cancer Res 56: 5522-5528.

5) Gillies RJ, Schornack PA, Secomb TW, Raghunand N (1999) Causes and effects of heterogeneous perfusion in tumors. Neoplasia 1: 197-207.

6) Gray LH, Conger AD, Ebert M, Hornsey S, Scott OC (1953) The concentration of oxygen dissolved in tissues at the time of irradiation as a factor in radiotherapy. Br J Radiol 26: 638-648.

7) Vaupel P (2004) The role of hypoxia-induced factors in tumor progression. The Oncologist 9(Suppl. 5), 10-17.

8) Vaupel P (2008) Hypoxia and aggressive tumor phenotype: implications for therapy and prognosis. The Oncologist 13: 21-26.

9) Wood JE, Litter J, Wilkins RW (1955) The mechanism of limb segment reactive hyperemia in man. Circ Res 3: 581-587.

10) Marcus M, Wright C, Doty D, Eastham C, Laughlin D, et al. (1981) Measurements of coronary velocity and reactive hyperemia in the coronary circulation of humans. Circ Res 49: 877-891.

11) Cohnheim J (1872) Untersuchungen ueber die embolische Processe. Berlin, A. Hirschwald.

12) Suzuki M, Hori K, Abe I, Saito S, Sato H (1981) A new approach to cancer chemotherapy: selective enhancement of tumor blood flow with angiotensin II. J Natl Cancer Inst 67: 663-669.

13) Peterson HI (1991) Modification of tumour blood flow-a review. Int J Radiat Biol 60: 201-210.

14) Sonveaux P, Jordan BF, Gallez B, Feron O (2009) Nitric oxide delivery to cancer: why and how? Eur J Cancer 45: 1352-1369.

15) Reyal J (2010) How could a Basic Knowledge of Vascular Physiology provide a New Tool for Tumor Oxygen-induced Radiosensitization- Postocclusive Reactive Hyperemia Concept for Synchronized Radiotherapy. WebmedCentral CANCER 1: WMC00604
16) Reyal J, Lebas N, Fourme E, Guihard T, Vilmer C, et al. (2012) Post-occlusive reactive hyperemia in basal cell carcinoma and its potential application to improve the efficacy of solid tumor therapies. Tohoku J Exp Med 227: 139-147.

17) Pettan-Brewer C, Morton J, Coil R, Hopkins H, Fatemie S, et al. (2012) B16 melanoma tumor growth is delayed in mice in an agedependent manner. Pathobiol Aging Age Relat Dis 2.

18) Roustit M, Millet C, Blaise S, Dufournet B, Cracowski JL (2010) Excellent reproducibility of laser speckle contrast imaging to assess skin microvascular reactivity. Microvasc Res 80: 505-511.

19) Baudelet C, Gallez B (2004) Effect of anesthesia on the signal intensity in tumors using BOLD-MRI: comparison with flow measurements by Laser Doppler flowmetry and oxygen measurements by luminescence-based probes. Magn Reson Imaging 22: 905-912.

20) Wen B, Urano M, O’Donoghue JA, Ling CC (2006) Measurements of partial oxygen pressure pO2 using the OxyLite system in R3327AT tumors under isoflurane anesthesia. Radiat Res 166: 512-518.

21) Stücker M, Springer C, Paech V, Hermes N, Hoffmann M, et al. (2006) Increased laser Doppler flow in skin tumors corresponds to elevated vessel density and reactive hyperemia. Skin Res Technol 12: 1-6.

22) Hill SA, Pigott KH, Saunders MI, Powell ME, Arnold S, et al. (1996) Microregional blood flow in murine and human tumours assessed using laser Doppler microprobes. Br J Cancer Suppl 27: S260263.

23) Ellingsen C, Ovrebø KM, Galappathi K, Mathiesen B, Rofstad EK (2012) pO2 fluctuation pattern and cycling hypoxia in human cervical carcinoma and melanoma xenografts. Int J Radiat Oncol Biol Phys 83: $1317-1323$.

24) Helfrich I, Scheffrahn I, Bartling S, Weis J, von Felbert V, et al. (2010) Resistance to antiangiogenic therapy is directed by vascular phenotype, vessel stabilization, and maturation in malignant melanoma. J Exp Med 207: 491-503.

25) Suzuki S, Heldin CH, Heuchel RL (2007) Platelet-derived growth factor receptor-beta, carrying the activating mutation D849N, accelerates the establishment of B16 melanoma. BMC Cancer 7: 224 .

Submit your manuscript to a JScholar journal and benefit from:

- Convenient online submission

ฯ Rigorous peer review

ๆ Immediate publication on acceptance

ब Open access: articles freely available online

ब High visibility within the field

- Better discount for your subsequent articles

Submit your manuscript at http://www.jscholaronline.org/submit-manuscript.php 